University of Nebraska - Lincoln

DigitalCommons@University of Nebraska - Lincoln

2020

\title{
Minimizing the duration of isolated attosecond pulses
}

Dian Peng

Anthony F. Starace

Hua-Chieh Shao

Jean Marcel Ngoko Djiokap

Follow this and additional works at: https://digitalcommons.unl.edu/physicsstarace

Part of the Atomic, Molecular and Optical Physics Commons, Elementary Particles and Fields and String Theory Commons, and the Plasma and Beam Physics Commons

This Article is brought to you for free and open access by the Research Papers in Physics and Astronomy at DigitalCommons@University of Nebraska - Lincoln. It has been accepted for inclusion in Anthony F. Starace Publications by an authorized administrator of DigitalCommons@University of Nebraska - Lincoln. 


\title{
Minimizing the duration of isolated attosecond pulses
}

\author{
Dian Peng (彭典), Anthony F. Starace, ${ }^{*}$ Hua-Chieh Shao, and Jean Marcel Ngoko Djiokap \\ Department of Physics and Astronomy, University of Nebraska, Lincoln, Nebraska 68588, USA
}

(Received 23 December 2019; accepted 7 December 2020; published 30 December 2020)

\begin{abstract}
We investigate theoretically how the duration of an isolated attosecond pulse (IAP) can be minimized by carefully selecting frequencies of high-order harmonic generation (HHG) spectra produced by ultrashort driving laser pulses. Based on numerical calculations of HHG by solving the time-dependent Schrödinger equation for a single $\mathrm{H}$ atom, we provide three strategies for generating shorter IAPs. First, when the high-frequency region of an HHG plateau is selected one should use frequencies below the cutoff. Second, for a wide HHG plateau the low-frequency region can produce shorter IAPs than the high-frequency region. Third, we propose a method of producing IAPs with transform-limited duration by selecting special frequency stripes across the entire HHG plateau. Analytic analyses show that how our strategies work is related to the (Fourier-transform) properties of an Airy function. We also carry out HHG calculations considering macroscopic effects by means of intensity averaging over the focal region. We find that our conclusions for single-atom calculations can still apply for macroscopic HHG spectra as long as they resemble single-atom spectra.
\end{abstract}

DOI: 10.1103/PhysRevA.102.063126

\section{INTRODUCTION}

Owing to the rich potentials that shorter laser pulses can bring to ultrafast-process research in a variety of systems [1-6], obtaining ever-shorter attosecond pulses has been a challenging quest in the field of attosecond science [7-9]. Besides recent direct optical methods [10], the most common way of generating attosecond pulses is to spectrally filter part of a high-order harmonic generation (HHG) spectrum [1-3]. If the driving laser pulse is long with many cycles, one can select a group of frequencies in the HHG spectrum to generate a train of attosecond pulses [11,12]. When the driving laser pulse is short with very few cycles, isolated attosecond pulses (IAPs) can be obtained by filtering a single region of the HHG spectrum [7-9,13-16]. IAPs of a few hundred attosecond (as) duration can be obtained by filtering the cutoff HHG [14,17], while the current shortest IAPs of sub-hundred-as duration are generated by filtering plateau frequencies below HHG cutoffs [7-9,18].

Generating shorter and stronger attosecond pulses has been the goal for many studies of ultrafast processes. For attosecond pulses that are generated from high-harmonic generation, enhancing HHG yield and increasing its frequency bandwidth are good approaches for producing attosecond pulses of higher intensities and shorter duration [19]. Another important aspect in generating shorter attosecond pulses is to overcome the attochirp problem. An attochirp is an intrinsic chirp of the HHG process where high-harmonic photons of different frequencies are emitted at different times [7]. As a consequence, the attosecond pulse is not transform limited, i.e., the duration is longer than the Fourier transform of the HHG spectrum bandwidth $[2,20]$. Previous works have demonstrated that a

*Deceased 5 September 2019. thin-film filter can partially compensate the attochirp for a limited frequency range $[16,21,22]$, or a shaped pulse waveform can reduce the intrinsic chirp during the HHG processes by squeezing returning electron trajectories into a short time window [23].

In this paper, we investigate how the duration of an isolated attosecond pulse can be minimized by carefully selecting frequencies of an HHG spectrum produced by linearly polarized one-cycle driving laser pulses without compensating the attochirp. Our studies are based on HHG spectra from an $\mathrm{H}$ atom which are computed by numerically solving the time-dependent Schrödinger equation (TDSE) [24] as well as by employing an analytic description of HHG produced by few-cycle pulses [25]. Our results show that (a) when the highfrequency region of an HHG plateau is selected one should use frequencies below the cutoff; (b) for a wide HHG plateau the low-frequency region can produce shorter IAPs than the high-frequency region; and (c) IAPs with transform-limited duration can be produced by selecting special frequency stripes across the entire HHG plateau.

Theoretical formulations of how HHG spectra and attosecond pulses are calculated for a single $\mathrm{H}$ atom are presented in Sec. II. Features of HHG spectra produced by one-cycle driving pulses and the accompanied carrier-envelope phase (CEP) issue are discussed in Sec. III A. Results of minimizing IAP duration for three frequency-selection categories are presented in Sec. III B for a single spectral range near the cutoff, Sec. IIIC for a single spectral range between cutoffs, and Sec. III D for a striped-frequency range (sequential but separated frequency regions or "stripes"). Section IV discusses how our strategies based on single-atom calculations behave when considering macroscopic effects of HHG. Analytic analyses about how our frequency-selection strategies work are presented in Sec. V. Lastly, Sec. VI summarizes our results and gives some conclusions. 


\section{THEORETICAL FORMULATION}

\section{A. Analytic calculation of HHG spectra}

The analytically calculated HHG spectrum $\rho(\Omega)$ is obtained by coherently adding half-cycle spectral amplitudes that correspond to electron trajectories ionized during each of the few half cycles of a laser pulse. For a detailed formulation of calculations, see Refs. [25,26]. In short, $\rho(\Omega)$ can be written as

$$
\rho(\Omega)=\sum_{j, k} s_{j k} \cos \left(\varphi_{j}-\varphi_{k}\right) \mathcal{A}_{j}(E) \mathcal{A}_{k}(E),
$$

where $\hbar \Omega$ is the photon energy, $j$ and $k$ label individual spectral amplitudes that originate from the $j$ th laser half cycle, $s_{j k}= \pm 1$ is a sign factor, $\varphi_{j}$ is the phase of the $j$ th amplitude, and $E$ is the electron return energy. Note that $\hbar \Omega=E+\left|E_{0}\right|$ with $E_{0}$ being the ground-state energy of the electron, which equals $E_{0}=-1 / 2$ a.u. (atomic units) for an $\mathrm{H}$ atom.

Each half-cycle spectral amplitude $\mathcal{A}_{j}(E)$ equals the square root of a product of three factors representing the three steps of HHG [27-30]: the ionization factor $\mathcal{I}_{j}$, the propagation factor $\mathcal{W}_{j}(E)$, and the recombination factor $\sigma^{(r)}(E)$ :

$$
\mathcal{A}_{j}(E) \equiv \sqrt{\mathcal{I}_{j} \mathcal{W}_{j}(E) \sigma^{(r)}(E)} .
$$

The $j$ th half-cycle spectral amplitude $\mathcal{A}_{j}(E)$ is determined by the electron trajectory that ionizes within laser half cycle $j$ and returns with the maximum energy. The ionization factor $\mathcal{I}_{j}$ is determined by the electric field at the time of tunneling ionization. The propagation factor $\mathcal{W}_{j}(E)$ is related to the electron excursion time in the laser field and an Airy function of energy $E$ which encodes the subcycle interference of short and long trajectories. The recombination factor $\sigma^{(r)}(E)$ is the photorecombination cross section of an electron to the $1 s$ ground state of an $\mathrm{H}$ atom.

\section{B. TDSE calculation of HHG spectra}

We also calculate HHG spectra by solving the TDSE for an $\mathrm{H}$ atom interacting with a laser field $F(t)$ that is linearly polarized along the $z$ axis:

$$
i \frac{\partial}{\partial t} \Psi(\boldsymbol{r}, t)=\left[\frac{p^{2}}{2}-\frac{1}{r}+z F(t)\right] \Psi(\boldsymbol{r}, t),
$$

where $m_{e},|e|$, and $\hbar$ are set to unity in atomic units. The TDSE is solved using a time-dependent generalized pseudospectral method [24], in which the wave function is expanded in Legendre polynomials and the time propagation is carried out using a second-order split-operator technique. The convergence of our TDSE calculations was monitored by increasing the basis size and the grid density in both space and time, where specific parameters are approximately $L \sim 200$ for the maximum angular momentum, $r_{\max } \sim 200$ a.u. for the maximum grid size, and $N \sim 1500$ for the number of radial grid points, and the time step is $\approx 0.125$ a.u.

The HHG spectrum $S(\Omega)$ is obtained from the Fouriertransformed dipole acceleration along the $z$ axis $\ddot{D}_{z}(\Omega)$ :

$$
S(\Omega)=\frac{1}{\hbar c^{3}}\left|\ddot{D}_{z}(\Omega)\right|^{2},
$$

where $c$ is the speed of light, the spectral (frequencydependent) dipole acceleration $\ddot{D}_{z}(\Omega)$ (or the spectral amplitude) is

$$
\ddot{D}_{z}(\Omega) \equiv \frac{1}{\sqrt{2 \pi}} \int_{-\infty}^{\infty} \ddot{D}_{z}(t) e^{i \Omega t} d t,
$$

and the time-dependent dipole acceleration $\ddot{D}_{z}(t)$ is [31]

$$
\begin{aligned}
\ddot{D}_{z}(t) & \equiv\langle\Psi(\boldsymbol{r}, t)|-\ddot{z}| \Psi(\boldsymbol{r}, t)\rangle \\
& =\left\langle\Psi(\boldsymbol{r}, t)\left|\frac{\partial V(r)}{\partial z}\right| \Psi(\boldsymbol{r}, t)\right\rangle+F(t),
\end{aligned}
$$

where $V(r)=-1 / r$ is the Coulomb potential for an $\mathrm{H}$ atom. Note that both the analytic spectrum $\rho(\Omega)$ and the TDSE spectrum $S(\Omega)$ are dimensionless in our formulations [25,32].

\section{Generating attosecond pulses}

Attosecond pulses are generated by spectrally filtering HHG spectra, i.e., by Fourier transforming the HHG spectral amplitude through a window function $w(\Omega)$. For the TDSE calculations, we define a field amplitude $d(t)$ as the inverse Fourier transform of the spectral dipole acceleration $\ddot{D}_{z}(\Omega)$ :

$$
d(t) \equiv \frac{1}{\sqrt{2 \pi}} \int_{-\infty}^{\infty} \ddot{D}_{z}(\Omega) w(\Omega) e^{-i \Omega t} d \Omega .
$$

For the analytic calculations, the spectrum $\rho(\Omega)$ can be written in a similar way as Eq. (4) [25], i.e.,

$$
\rho(\Omega)=\frac{\Omega^{4}}{\hbar c^{3}}|D(\Omega)|^{2},
$$

where $D(\Omega)$ is the Fourier-transformed dipole moment. In this case, the field amplitude $d(t)$ is defined as

$$
d(t) \equiv \frac{1}{\sqrt{2 \pi}} \int_{-\infty}^{\infty} \Omega^{2} D(\Omega) w(\Omega) e^{-i \Omega t} d \Omega .
$$

The attosecond pulse intensity profile $I(t)$ is then proportional to the modulus square of the field amplitude:

$$
I(t)=I_{0}|d(t)|^{2} .
$$

The pulse duration $\Delta t$ is defined as the full width at half maximum (FWHM) of the intensity profile $I(t)$. Since the constant $I_{0}$ does not affect the pulse duration $\Delta t$ or the comparison of relative pulse intensities, we set $I_{0}=1$ with dimensions $1 / \hbar c^{3} \omega_{0}^{2}$ so that $I(t)$ is treated dimensionlessly.

For ease of calculations, we use a rectangular window function $w(\Omega)$ to select HHG frequencies:

$$
w(\Omega)=\left\{\begin{array}{ll}
1 & \Omega \in \text { selected frequency range } \\
0 & \text { otherwise }
\end{array} .\right.
$$

A smooth window function such as a super-Gaussian function with a flat top will not affect our attosecond pulse results much. Note that, unlike studies in Ref. [33], we do not compensate phases in HHG spectra during frequency selections using Eq. (11).

\section{RESULTS FOR SINGLE-ATOM CALCULATIONS}

\section{A. HHG spectra produced by one-cycle laser}

In order to generate isolated attosecond pulses instead of pulse trains, the effective driving pulse duration must be short 

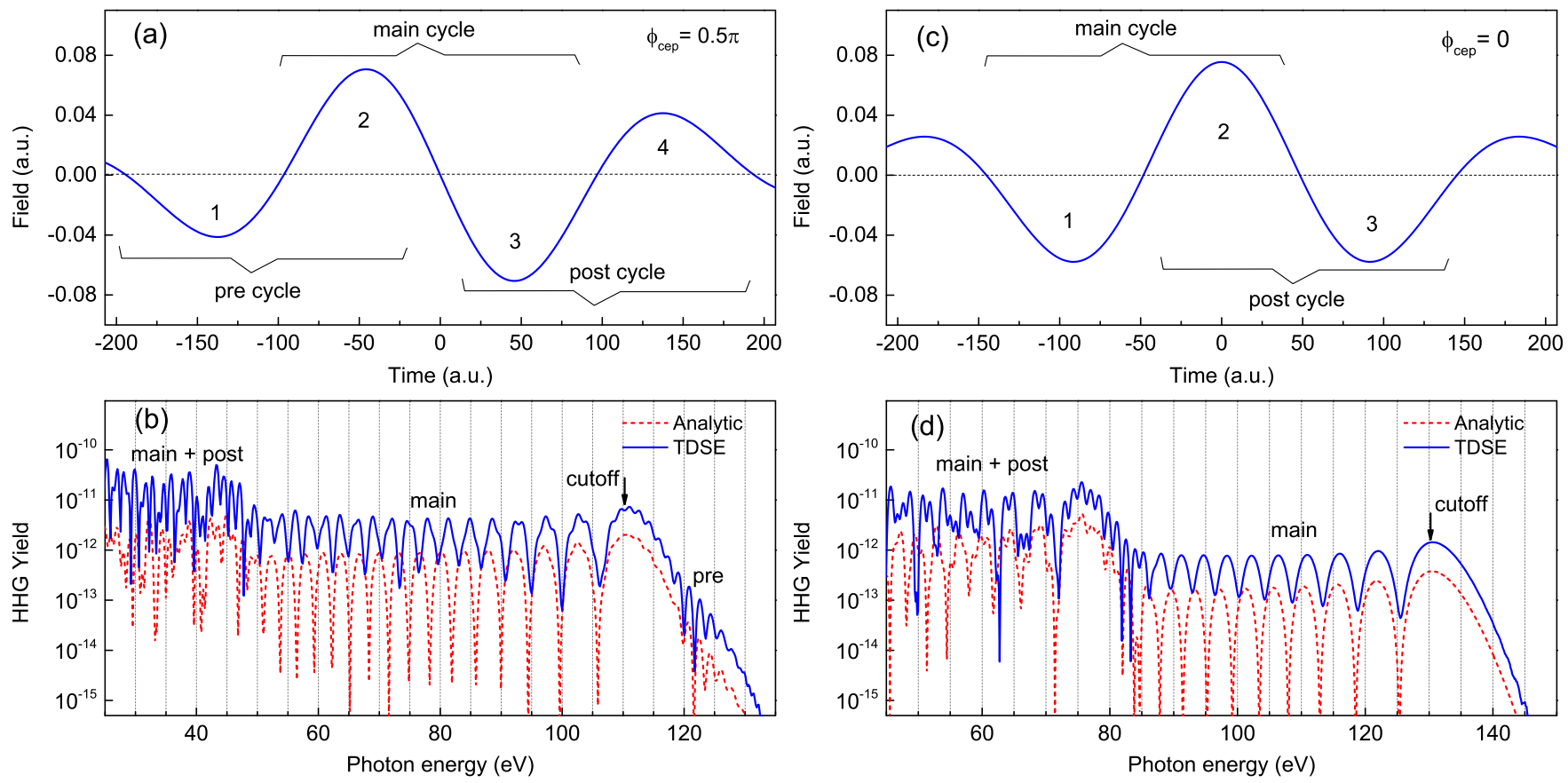

FIG. 1. (a) The electric field $F(t)$ of a one-cycle sine-shaped Gaussian pulse with a carrier wavelength of $1500 \mathrm{~nm}$ and a peak intensity of $2 \times 10^{14} \mathrm{~W} / \mathrm{cm}^{2}$. (b) Calculated HHG spectra produced by the laser pulse in panel (a) from an $\mathrm{H}$ atom. The solid line is obtained by TDSE calculations and the dashed line is obtained by analytic calculations. (c) Same as panel (a) except for the CEP which gives a cosine-shaped pulse. (d) Calculated HHG spectra produced by the laser field in panel (c). See text for labeling explanations.

such that only one laser cycle contributes to the target HHG spectrum $[2,15,16]$. Therefore, we use a one-cycle Gaussian pulse as our driving laser field. Specifically, for a linearly polarized electric field, its vector potential magnitude $A(t)$ is described as

$$
A(t)=-\frac{c F_{0}}{\omega_{0}} \exp \left(-\frac{2 \ln 2}{\tau^{2}} t^{2}\right) \sin \left(\omega_{0} t+\phi_{\text {cep }}\right),
$$

where $F_{0}$ is the peak amplitude, $\omega_{0}$ is the carrier frequency, $\tau$ is the pulse duration, and $\phi_{\text {cep }}$ is the carrier-envelope phase. A one-cycle pulse has a FWHM in the intensity of $\tau=2 \pi / \omega_{0}$. The electric field is then obtained from the time derivative of the vector potential, i.e.,

$$
F(t)=-\frac{1}{c} \frac{\partial A(t)}{\partial t} .
$$

The spatial dependence of the laser field is neglected because the electric dipole approximation is applicable.

In order to generate short attosecond pulses, we would like broad HHG spectra with large cutoff energies. Based on the classical cutoff formula [27-29], two effective ways of obtaining large HHG cutoff energies are to use high intensities and long wavelengths of the driving laser field. For an $\mathrm{H}$ atom, we choose in our calculations a laser peak intensity of $2 \times 10^{14} \mathrm{~W} / \mathrm{cm}^{2}$, which gives a peak amplitude $F_{0}=0.076$ a.u., and a carrier wavelength of $1500 \mathrm{~nm}\left(=2 \pi c / \omega_{0}\right)$.

For short driving pulses, the CEP is important [34-36]. Figure 1 shows the electric fields of a sine- and cosine-shaped one-cycle pulse and the HHG spectra produced from those two fields. One sees that, for a one-cycle driving laser, the cosine-shaped pulse in Fig. 1(c) gives a higher cutoff energy [25,34]: the 130-eV cutoff in Fig. 1(d) compared to the
110-eV cutoff in Fig. 1(b). Here, the cutoffs correspond to the maximum return energies of the electron plus the ionization potential. On the other side, the sine-shaped pulse in Fig. 1(a) gives a wider HHG plateau that comes from a single laser cycle: the "main" plateau in Fig. 1(b) has a bandwidth of $62 \mathrm{eV}$ compared to that of $47 \mathrm{eV}$ in Fig. 1(d). A wider singlecycle plateau is more favorable than the higher cutoff energy for generating short isolated attosecond pulses. Thus we use the sine-shaped pulse, i.e., $\phi_{\text {cep }}=0.5 \pi$ in Eq. (12), for our following studies on attosecond pulse generation.

The electric field of a one-cycle sine-shaped pulse, plotted in Fig. 1(a), contains four half cycles, labeled as 1 through 4 in the figure. These four half cycles form three full cycles: the precycle ( 1 and 2$)$, the main cycle ( 2 and 3 ), and the postcycle (3 and 4). HHG spectra produced by such a pulse are presented in Fig. 1(b), which are calculated from both analytic and TDSE methods. One sees that the analytic and TDSE spectra are very similar besides an overall intensity difference [26]. In general, HHG spectra obtained from the analytic method are accurate near the cutoff, and compare well with TDSE results over the entire plateau region. Moreover, this analytic method provides clear physical interpretations that we have previously utilized to explore how HHG can be enhanced by time delays [26] or chirps [32] in a two-color (e.g., $\omega-2 \omega)$ setup. The same interpretations are used in the following to explain the trajectory correspondence of HHG spectra.

The HHG spectrum shown in Fig. 1(b) exhibits a twoplateau structure. The high-energy (main) plateau, above $48 \mathrm{eV}$ with a cutoff of $110 \mathrm{eV}$, comes from electron motion during the main cycle of the laser pulse, i.e., the electron wave packet ionizes during the half cycle 2 and returns within one 
cycle after ionization. The low-energy plateau, below $48 \mathrm{eV}$ with a cutoff of $44 \mathrm{eV}$, is due to mainly electron motion during the postcycle of the laser field, i.e., the electron wave packet ionizes during the half cycle 3 and returns within one cycle after ionization. The more irregular structure of the lowerenergy plateau compared to the higher-energy plateau is due to the fact that electron motions during both the main and postcycles contribute to this energy range and they interfere. Electron motion during the precycle contributes negligibly to the HHG spectrum owing to low ionization from the half cycle 1 , which forms modulations around $120 \mathrm{eV}$ with an intensity that is two orders of magnitude lower than the main plateau.

Similarly, the HHG spectrum in Fig. 1(d) produced by a cosine-shaped one-cycle pulse in Fig. 1(c) also presents two plateaus: a high-energy (main) plateau above $82 \mathrm{eV}$ with a cutoff of $130 \mathrm{eV}$ and a low-energy plateau below $82 \mathrm{eV}$ with a cutoff of $75 \mathrm{eV}$. Both cutoff energies in Fig. 1(d) are higher than the cutoff energies in Fig. 1(b). This is because the electric fields of half cycles 2 and 3 in Fig. 1(c) are stronger than the electric fields of half cycles 3 and 4 in Fig. 1(b), and ionized electrons gain more energy during those returning half cycles with stronger electric fields. The bandwidth of the main plateau in Fig. 1(d) is narrower than that of Fig. 1(b). This is because the increase in cutoff energies for the postcycle is larger than that for the main cycle, which is due to the more pronounced field enhancement between half cycle 3 in Fig. 1(c) and half cycle 4 in Fig. 1(a) as compared to the field enhancement between half cycle 2 in Fig. 1(c) and half cycle 3 in Fig. 1(a).

We use the high-energy (main) plateau of the HHG spectrum in Fig. 1(b) to generate isolated attosecond pulses, since only one laser cycle contributes to this HHG spectrum range. Notice that for the high-energy plateau, there are large oscillations with a varying energy spacing between neighboring peaks. These oscillations originate from subcycle interference of short and long trajectories during the main cycle of the laser field, and they can be described analytically by squaring an Airy function [25,37]. Thus, we call these oscillations Airy peaks to distinguish them from harmonic peaks which come from intercycle interference and present an equal spacing of $2 \omega_{0}$ between neighboring peaks. Airy peaks are observed in many HHG spectra produced by few-cycle driving lasers for single-atom calculations $[25,33,34,38,39]$. Note that the key to obtain Airy peaks is that only one laser cycle contributes dominantly to the HHG spectrum, which does not require strictly a single-cycle laser pulse. For example, our calculations show that a $20 \%$ longer driving pulse still gives a nice wide spectrum with many Airy peaks.

\section{B. Results for a single spectral range near cutoff}

One common way of generating isolated attosecond pulses is to filter a frequency bandwidth near the cutoff of an HHG spectrum [14,17]. In this section, we show that based on single-atom HHG calculations the selected frequency bandwidth should not expand across the cutoff in order to produce short IAPs. Figure 2 shows attosecond pulses generated when selecting a single spectral range the coverage of which is either below the cutoff, 90-106 and 90-110 eV, or across the cutoff, $90-120 \mathrm{eV}$, where the cutoff is $110 \mathrm{eV}$. One sees that

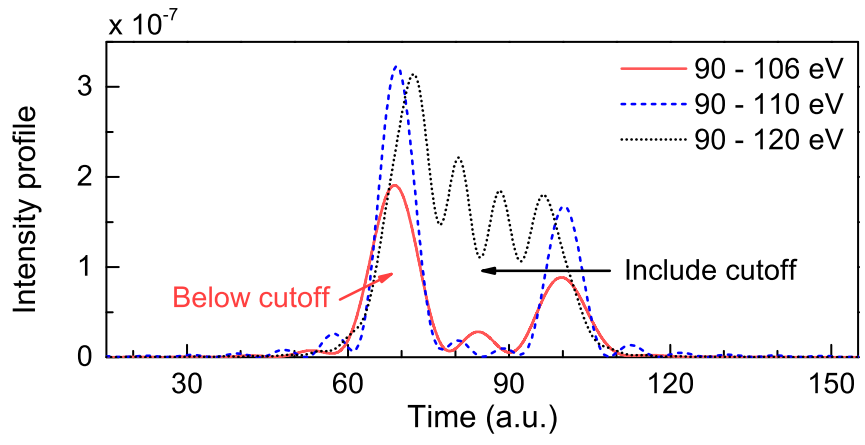

FIG. 2. Pulses generated from a single spectral range of the TDSE HHG spectrum in Fig. 1(b). The bandwidth range is either below (90-106 and 90-110 eV) or across (90-120 eV) the cutoff at $110 \mathrm{eV}$. The time scale is that of Fig. 1(a).

when the frequency range lies below the cutoff, a pair of short pulses is generated corresponding to short and long trajectory emissions. However, when the frequency bandwidth includes the cutoff peak, a long pulse is generated with subpeak structures. Therefore, in order to obtain short IAPs, the selected frequency range should lie below the cutoff.

The cross-cutoff behavior of attosecond pulses from singleatom HHG calculations, i.e., the sudden change of the pulse structure such that either a pair of short pulses or a long pulse is generated depending on whether the selected frequency range includes the cutoff, is due to the fact that the cutoff Airy peak is spectrally much wider than other Airy peaks. More discussions through analyses by manually varying the width of the cutoff Airy peak are presented in Sec. V B.

\section{Results for a single spectral range between cutoffs}

In this section, we investigate attosecond pulses generated by selecting harmonic frequencies of a single spectral range between HHG cutoffs. The HHG spectrum in Fig. 1(b) has two plateaus with two cutoffs: the lower-energy plateau with a cutoff of $44 \mathrm{eV}$ and the higher-energy plateau with a cutoff of $110 \mathrm{eV}$. We select a range of frequencies between these two cutoffs, i.e., between 44 and $110 \mathrm{eV}$, to generate attosecond pulses.

It is known that the attosecond pulses are not transform limited, i.e., the duration is not as short as the Fourier transform of the HHG spectrum bandwidth, owing to the attochirp [20]. For example, when selecting a narrow frequency bandwidth that covers a portion of the plateau, i.e., $60-90 \mathrm{eV}$, a pair of short pulses is generated, as shown in Fig. 3(a). However, when selecting a wide frequency bandwidth that covers the whole plateau, i.e., $50-110 \mathrm{eV}$, pulses are much longer with subpeaks. The longer pulse is due to the attochirp which originates from the fact that electrons with different energies return at different times. Figure 3(b) shows the electron return time as a function of its return energy from classical calculations. The blue horizontal line indicates the division between short and long trajectories, where the cutoff trajectory returns at time $t_{r}\left(E_{\max }\right)=85.5$ a.u. with a maximum energy of $110 \mathrm{eV}$. Comparing the emission times of attosecond pulses in Fig. 3(a) with the return times from classical trajectories, it is clear that the earlier one of the pulse pair, located 

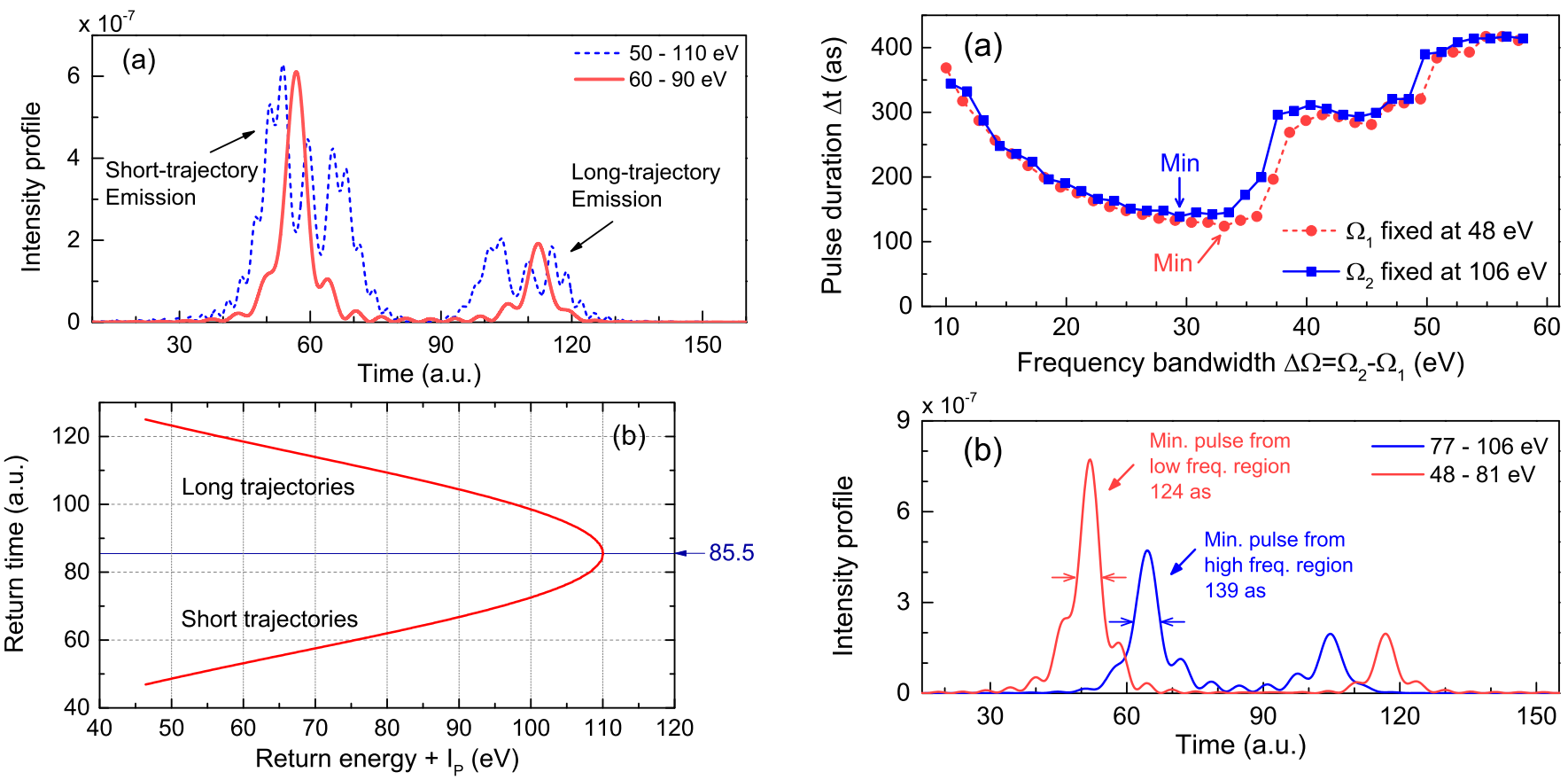

FIG. 3. (a) Attosecond pulses generated from a single spectral range of the TDSE HHG spectrum in Fig. 1(b). The solid red line shows pulses generated from frequencies of $60-90 \mathrm{eV}$, and the dashed blue line shows pulses generated from a doubled frequency bandwidth of 50-110 eV. (b) Electron return time as a function of its return energy from classical calculations. Time scales are the same as that in Fig. 1(a). See text for labeling explanations.

around 60 a.u., corresponds to short trajectory emissions, and the later one, located around 110 a.u., corresponds to long trajectory emissions $[11,38]$. Short trajectory emissions are often selected in experiments [7,8,11], and thus we shall focus on the earlier pulse for the rest of this section.

For a wide HHG plateau such that only part of the spectrum can be used to generate short attosecond pulses due to the attochirp, we investigate which part of the HHG spectrum one should use to minimize the duration of IAPs. For this purpose, we perform a bandwidth scan to see how the pulse duration behaves as the frequency bandwidth increases.

We set a single spectral range of $\left[\Omega_{1}, \Omega_{2}\right]\left(\Omega_{1}<\Omega_{2}\right)$ with a frequency bandwidth $\Delta \Omega \equiv \Omega_{2}-\Omega_{1}$. In this case, the window function $w(\Omega)$ is

$$
w(\Omega)=\left\{\begin{array}{ll}
1 & \Omega_{1} \leqslant \Omega \leqslant \Omega_{2} \\
0 & \text { otherwise }
\end{array} .\right.
$$

In order to reveal properties in different energy regions of the spectrum, we carry out the bandwidth scan starting from two opposite sides. First, we fix $\Omega_{1}$ at the lowest-energy end of the plateau $\left(\Omega_{1}=48 \mathrm{eV}\right)$ while $\Omega_{2}$ is scanned from low energies to high energies in the spectrum until $\Omega_{2}$ reaches $106 \mathrm{eV}$. Then, we reverse the scan, i.e., fixing $\Omega_{2}=106 \mathrm{eV}$ and scanning $\Omega_{1}$ from high energies to low energies. For both cases, we record the pulse duration $\Delta t$ from the short trajectory emission as the frequency bandwidth $\Delta \Omega$ increases, and the results are plotted in Fig. 4(a). Note that as the frequency bandwidth gets wider, the attosecond pulses exhibit subpeak structures, as shown in Fig. 3(a) for the wider bandwidth. For

FIG. 4. (a) Plots of attosecond pulse duration $\Delta t$ as a function of the frequency bandwidth $\Delta \Omega$ for a single spectral range between cutoffs. Dash-dot symbols are for the bandwidth scan when $\Omega_{1}$ is fixed at $48 \mathrm{eV}$ and line-square symbols are for the bandwidth scan when $\Omega_{2}$ is fixed at $106 \mathrm{eV}$. The minimum pulse duration for each case is indicated by arrows. (b) Minimum pulses as indicated by arrows in panel (a) that are generated from low- and high-frequency regions and are plotted on the same time scale as Fig. 1(a).

these pulses with subpeak structures, the pulse duration $\Delta t$ is the width of the entire single pulse.

A common feature for the two cases in Fig. 4(a) is that, as the frequency bandwidth $\Delta \Omega$ increases, the pulse duration $\Delta t$ first decreases, then reaches a limit and rises up rapidly. However, when $\Omega_{1}$ is fixed on the low-energy end, the minimum pulse duration is 124 as with a bandwidth of $33 \mathrm{eV}$, whereas when $\Omega_{2}$ is fixed on the high-energy end, the minimum pulse duration is 139 as with a bandwidth of $29 \mathrm{eV}$. Thus, the low-frequency region of the spectrum can produce shorter attosecond pulses than the high-frequency region [40].

The minimum pulses generated from low-frequency and high-frequency regions are shown in Fig. 4(b). One sees that besides the shorter pulse duration, the minimum pulse obtained from the low-frequency region also has a higher peak intensity than the minimum pulse obtained from the highfrequency region. Emission times of the pulse pair generated from low frequencies are farther apart compared to that of high frequencies [21]. This can be understood by the electron return times shown in Fig. 3(b): Electrons with lower return energies have a bigger difference in return times between short and long trajectories.

\section{Results for a striped-frequency range}

In this section, we present a way of producing short isolated attosecond pulses. This method selects sequential but separated frequency regions (stripes) across the entire HHG spectrum and generates isolated attosecond pulses with 
transform-limited pulse duration without compensating the attochirp. Specifically, for the HHG spectrum in Fig. 1(b), we select every other Airy peak in the high-energy plateau and generate a single short pulse from these frequency stripes, or a striped-frequency pulse (SFP).

The high-energy plateau of the TDSE HHG spectrum in Fig. 1(b) is replotted in Fig. 5(a) on a linear scale. There are 19 Airy peaks, labeled 1 starting from the cutoff peak at $110 \mathrm{eV}$, through 19 for the lowest-energy peak at $49.5 \mathrm{eV}$. As an example, we select all the odd Airy peaks (1, 3, 5, etc., through 19) and the generated pulse is plotted in Fig. 5(c). The pulse duration $\Delta t$, defined as the FWHM of the pulse intensity profile described in Eq. (10), is indicated in the figure. One sees that a single pulse is obtained at the center, i.e., at time $t_{r}\left(E_{\max }\right)=85.5$ a.u., with negligible sidebands. One can also select even Airy peaks (2, 4, 6, etc., through 18) to generate a SFP as shown in Fig. 5(d). The even-peak SFP has a longer duration of 57 as compared to 45 as of the odd-peak pulse because the frequency bandwidth of even Airy peaks is smaller than that of odd Airy peaks. Both SFPs, i.e., from either odd or even Airy peaks, are shorter than the minimum pulse generated by selecting a single spectral range, the pulse duration of which is 124 as. The odd-peak SFP is also more intense than the minimum pulse generated by selecting a single spectral range.

The duration of striped-frequency pulses is very close to that of the transform-limited pulse from a square spectrum, as shown in Fig. 5(b). A square spectrum can be obtained by simply setting $\ddot{D}_{z}(\Omega)=1$ in Eq. (7) and taking a rectangular window function like Eq. (14). The bandwidth-duration product of the transform-limited pulse from a square spectrum is $\Delta \Omega \Delta t=3664 \mathrm{eV}$ as $=5.566$ a.u. For a striped-frequency range of odd Airy peaks, we start with peak 1 and gradually add additional lower-energy peaks. The frequency bandwidth and corresponding pulse duration are recorded and plotted in Fig. 5(b). The bandwidth for a striped-frequency range is defined as its frequency coverage, i.e., the energy difference between the left and right ends of selected peaks. For example, the frequency bandwidth $\Delta \Omega$ for selecting all the odd peaks is $\Delta \Omega=120-48=72 \mathrm{eV}$.

One sees from Fig. 5(b) that the duration of stripedfrequency pulses produced from odd Airy peaks is very close to the transform-limited pulse duration for a square spectrum. The discrepancy of the small bandwidth point is an artifact of the bandwidth definition for a striped-frequency range: The spectral shape is no longer like a square for a small bandwidth. The duration of SFPs produced from only even Airy peaks (not shown in the figure) is also very close to the transformlimited pulse duration for a square spectrum.

The mechanism of generating SFPs can be understood by the phase structure of the HHG spectrum. Adjacent peaks in the spectrum have an approximate $\pi$ difference in the spectral phase. Selecting every other peak means selecting only the positive (or negative) parts of the spectral amplitude. This results in a pulse peak generated at time $t_{r}\left(E_{\max }\right)$. Moreover, the attochirp in the HHG spectrum suppresses sidebands that are also generated from selecting every other peak. Detailed discussions through coherent summation of field amplitudes generated from individual Airy peaks are presented in Sec. V C.
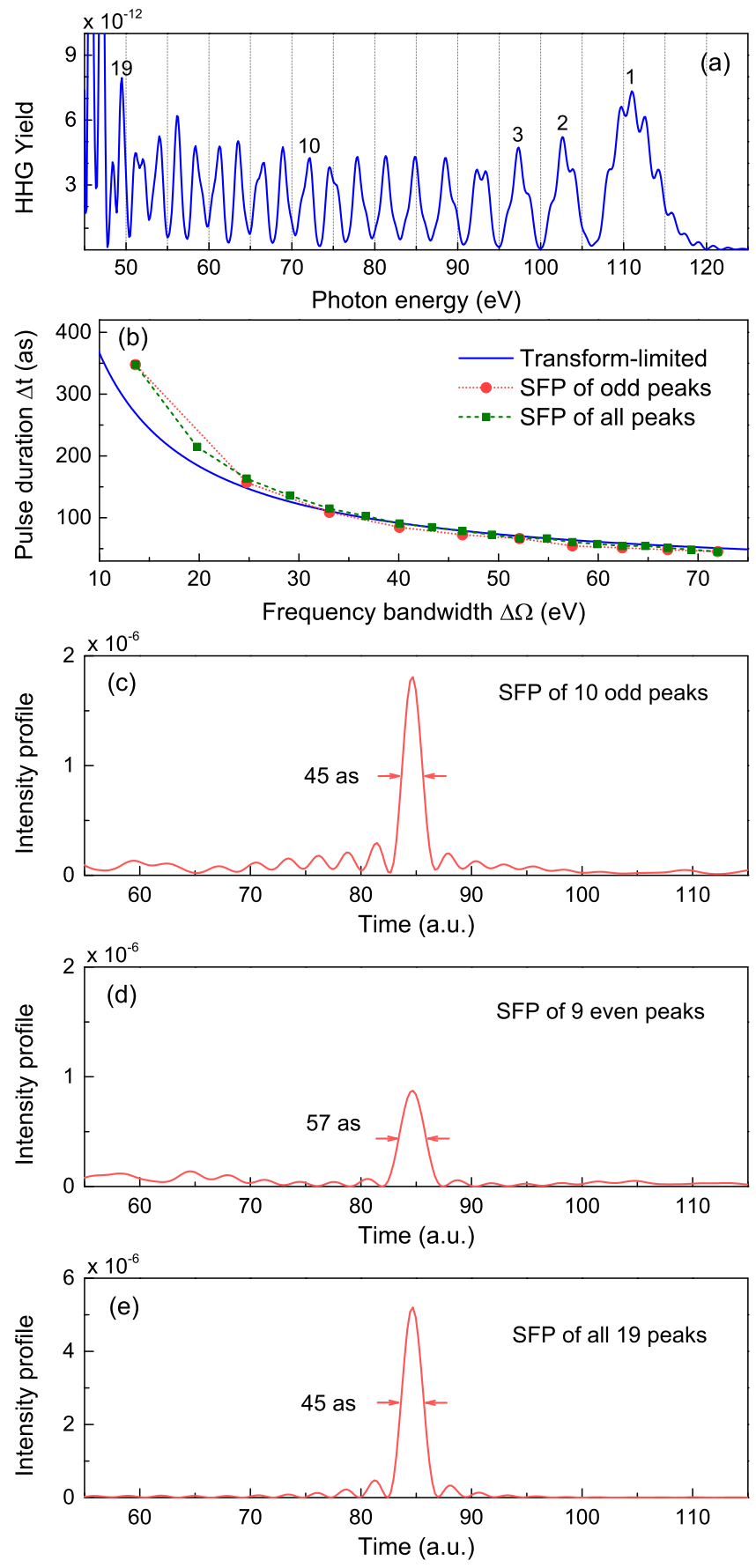

FIG. 5. (a) Linear replot of the TDSE HHG spectrum in Fig. 1(b). Numbers label Airy peaks. (b) Plots of pulse duration $\Delta t$ as a function of the frequency bandwidth $\Delta \Omega$ for the transform-limited case of a square spectrum (solid line), the striped-frequency pulse (SFP) case of odd Airy peaks (dot-line symbols), and the SFP case of all peaks (square-dash symbols). (c-e) Striped-frequency pulses from all odd peaks, all even peaks, and all peaks of the HHG spectrum. The time scale is that of Fig. 1(a).

Since adjacent Airy peaks in the HHG spectrum have an approximate $\pi$ difference in the spectral phases, we can improve our SFP method by introducing a phase $\pi$ to even Airy peaks and select both odd and even peaks to generate stronger isolated attosecond pulses. Figure 5(e) shows an improved 


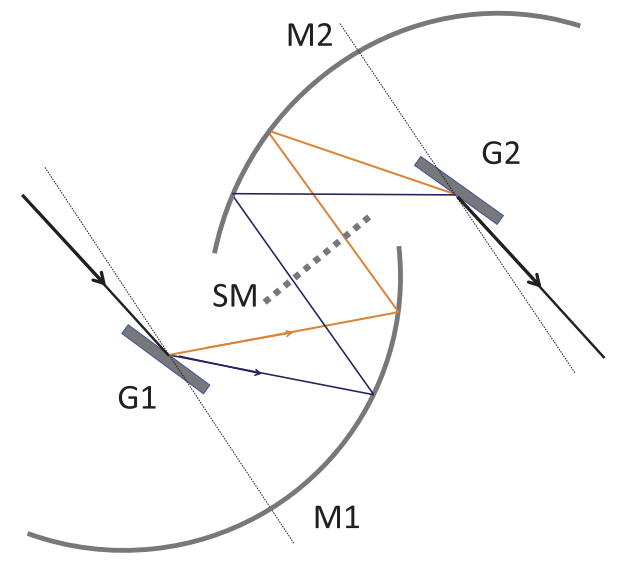

FIG. 6. Illustration of a dispersion-free optical setup for selecting frequency stripes of every other Airy peak in the HHG spectrum. G1 and G2 are soft-x-ray gratings. M1 and M2 are parabolic mirrors. SM is a spatial mask to block unwanted frequencies. Gratings are placed at the focuses of mirrors.

SFP when all Airy peaks are included and a phase $\pi$ added to even peaks. Owing to constructive interference of both odd and even Airy peaks, the improved SFP exhibits a much stronger peak intensity compared to regular SFPs from either odd or even Airy peaks. The pulse duration of improved SFPs remains at the transform-limited level, as shown in Fig. 5(b).

One possible way to select frequency stripes of every other Airy peak in the HHG spectrum is a dispersion-free $4 f$ setup illustrated in Fig. 6, which resembles the configuration of a zero-dispersion pulse compressor and a pulse shaping mask used in femtosecond pulse shaping for infrared and visible light [41]. The soft-x-ray beam produced from HHG is first dispersed by a grating G1 (e.g., Shimadzu, 1200 1/mm [9]), and then filtered by a structured spatial mask SM so that unwanted frequencies are blocked (or alternatively selected frequencies are changed in phase by $\pi$ ), and finally a second grating G2 combines remaining Airy-peak frequencies and generates a SFP. Parabolic mirrors are located at one focal length from the gratings and from the spatial mask. This setup ensures no dispersion, i.e., equal paths for different frequencies. For good reflectivity of a broad bandwidth, one may use diamondlike carbon coated mirrors with grazing incidence [9] which is not illustrated in Fig. 6.

\section{RESULTS FOR FOCAL AVERAGING}

We have shown how the duration of isolated attosecond pulses can be minimized by carefully selecting frequencies of a one-cycle HHG spectrum based on single-atom HHG calculations. Such spectra exhibit Airy peaks, which are large oscillations with uneven widths [25,33,34,38,39]. Experimental HHG spectra from few-cycle laser pulses can exhibit a supercontinuum structure $[8,9]$. Large scale modulated structures around the cutoff accompanied with fine oscillations in lower energies are also observed in many experiments [42-44]. In this section, we investigate how macroscopic effects of HHG play a role in minimizing the duration of IAPs by taking account of the longitudinal and transverse averaging of the driving laser spatial profile.
We take the integral approach to calculate the macroscopic effects where local high-harmonic radiations within the gas medium are integrated over the interaction region with proper phases [45]. We consider a dilute gas and low ionization where absorption of high-harmonic radiation and distortion of the driving pulse can be ignored [11]. We also consider a gently focused laser beam with a narrow gas target (compared to the laser Rayleigh length) so that the laser intensity does not vary much along the gas medium. We estimate the propagation effects for two representative situations.

In the first case, we consider the interaction region as one long thin tube, where the laser intensity variance is small so that we ignore key phase factors coming from Gouy phase (induced by focusing of the driving laser) and dipole phase (linearly dependent on laser intensity) [46]. Thus, threedimensional (3D) propagation is reduced to a one-dimensional (1D) averaging with good phase matching in the forward direction. Results for 1D intensity averaging are presented in Sec. IV A. Note that this averaging still takes into account coherence of short and long trajectories.

In the second case, we consider the interaction region as a bunch of many long thin tubes where coherence inside tubes is considered but coherence between tubes is ignored. We test the plausibility of this 3D modeling by comparing our calculation with results in Ref. [47] where fully 3D propagation is considered, and we see good qualitative agreements. Results for 3D intensity averaging are presented in Sec. IV B, where we estimate HHG spectra at the medium exit by integrating over a cylindrical volume, which consists of a 1D coherent intensity averaging along the propagating axis [48,49] and a two-dimensional incoherent intensity averaging perpendicular to the axis [11].

\section{A. One-dimensional intensity averaging}

We consider a gently focused Gaussian laser beam with a narrow gas target (compared to the laser Rayleigh length) so that the laser intensity does not vary much along the gas medium. For example, considering a driving wavelength of $1500 \mathrm{~nm}$ and a beam waist of $50 \mu \mathrm{m}$, the laser intensity drop is less than $4 \%$ for a 1-mm-long gas medium near the focus. For a uniform gas density, we estimate phase-matched highharmonic radiation at the medium exit by coherently summing HHG amplitudes produced by a range of laser peak intensities $[48,49]$ :

$$
S_{1 \mathrm{D}}(\Omega)=\frac{1}{\hbar c^{3}}\left|\sum_{i} \ddot{D}_{z}^{(i)}(\Omega)\right|^{2},
$$

where $\ddot{D}_{z}^{(i)}(\Omega)$ is the spectral amplitude corresponding to the $i$ th laser intensity. Specifically, we choose the peak intensity range from 1.96 to $2.04 \times 10^{14} \mathrm{~W} / \mathrm{cm}^{2}$, and the number of $i$ 's is chosen such that the HHG spectrum is converged. Attosecond pulses are generated by Fourier transforming the total HHG spectral amplitudes through a window function.

The calculated TDSE HHG spectrum with 1D intensity averaging is shown in Fig. 7. Compared with the single-atom HHG spectrum in Figs. 1(b) and 5(a), the ordinary HHG harmonics with an equal spacing of $2 \omega_{0}$ in the low-energy plateau are well resolved (see the spectrum below $50 \mathrm{eV}$ in the inset of 


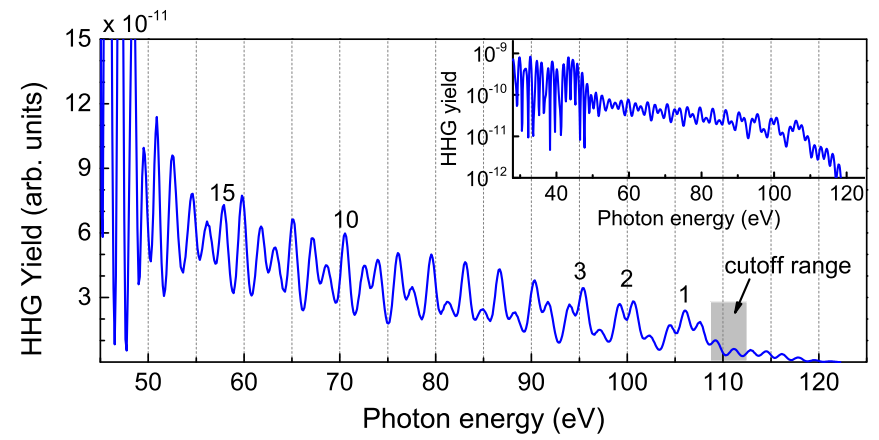

FIG. 7. TDSE HHG spectrum with 1D intensity averaging calculated using Eq. (15). Numbers label Airy peaks. The inset shows the same spectrum for a broader energy range with a log scale.

Fig. 7), while the Airy peaks in the high-energy plateau above $50 \mathrm{eV}$ have more complex structures due to the interference of spectral amplitudes from different laser intensities. The cutoff range corresponding to the laser peak intensities is 108.7 to $112.5 \mathrm{eV}$, which is indicated in the figure.

For a single spectral range near the cutoff, we select a frequency bandwidth that is either below the cutoff region, 90-109 eV, or across the cutoff region, 90-120 eV, and the generated attosecond pulses are plotted in Fig. 8. Unlike the attosecond pulses generated from the single-atom HHG spectrum in Fig. 2 where both short and long trajectory emissions are significant, the long trajectory emission in Fig. 8 around time 100 a.u. is negligible compared to the short trajectory emission around time 70 a.u. due to the intensity averaging [50]. When the bandwidth is below the cutoff range, the duration of the attosecond pulse is 193.5 as; when the bandwidth includes the cutoff range, the duration of the attosecond pulse is longer with 205.6 as. Since the HHG yield of the cutoff range is much lower than that below the cutoff range, the effect of including the cutoff range is less pronounced compared to that for the single-atom case.

For a single spectral range between cutoffs, we carry out similar bandwidth scans as in Sec. III C with either $\Omega_{1}$ fixed at the low energy end of $49 \mathrm{eV}$ or $\Omega_{2}$ fixed at the high energy end below the cutoff region of $109 \mathrm{eV}$. One sees in Fig. 9(a) that the pulse duration of the high-frequency region starts to rise when the bandwidth reaches $27 \mathrm{eV}$, while the pulse dura-

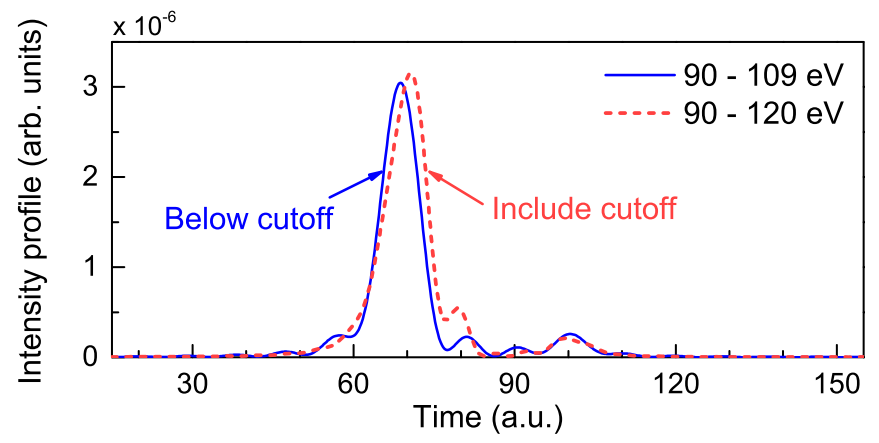

FIG. 8. Attosecond pulses for a single spectral range that either lies below $(90-109 \mathrm{eV})$ the cutoff range or includes $(90-120 \mathrm{eV})$ the cutoff range.
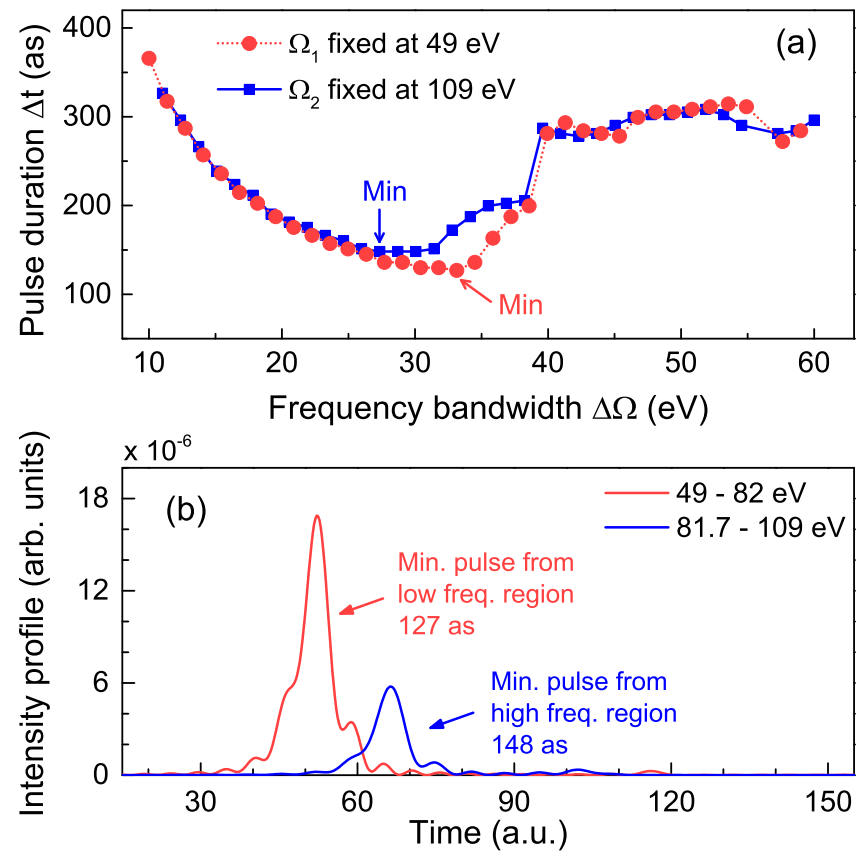

FIG. 9. (a) Plots of attosecond pulse duration $\Delta t$ as a function of the frequency bandwidth $\Delta \Omega$ for two bandwidth scans. Dash-dot symbols are for the case when $\Omega_{1}$ is fixed at $49 \mathrm{eV}$. Line-square symbols are for the case when $\Omega_{2}$ is fixed at $109 \mathrm{eV}$. The minimum pulse duration for each case is indicated by arrows. (b) Minimum pulses that are generated from low- and high-frequency regions and are plotted on the same time scale as Fig. 1(a).

tion of the low-frequency region rises at a larger bandwidth of $33 \mathrm{eV}$. The minimum attosecond pulses generated from both regions are plotted in Fig. 9(b) where the low-frequency pulse has a bandwidth of $49-82 \mathrm{eV}$ and the high-frequency pulse has a bandwidth of $82-109 \mathrm{eV}$. Besides the shorter duration of 127 as as compared to 148 as, the minimum pulse from the low-frequency region is also more intense than that from the high-frequency region due to the higher HHG yield in the low-frequency region.

For a striped-frequency range, we first select eight odd peaks $(1,3,5$, etc., through 15) and the generated attosecond pulse is plotted in Fig. 10(a). One sees that a short pulse is produced around time 86 a.u. but with significant side pulses at earlier times. To suppress those side pulses, we employ the improved SFP method where both odd and even peaks are selected with a phase $\pi$ added to even peaks. One sees in Fig. 10(b) that the improved SFP of all 15 peaks produces a single short pulse of 63.5 as with significantly suppressed sidebands. Compared to the SFP of odd peaks, the improved SFP produces weaker sidebands and a stronger main pulse. Figure 10(c) plots the pulse duration of the improved SFPs when the number of peaks included increases from 1 to 15 . Clearly, the pulse duration of improved SFPs still remains at the transform-limited level. Note that when more than 15 peaks are included, the pulse duration of improved SFPs increases slightly and this is the reason we include only up to 15 peaks.

Striped-frequency pulses need Airy peaks of one-cycle HHG spectra which originate from subcycle interference of 

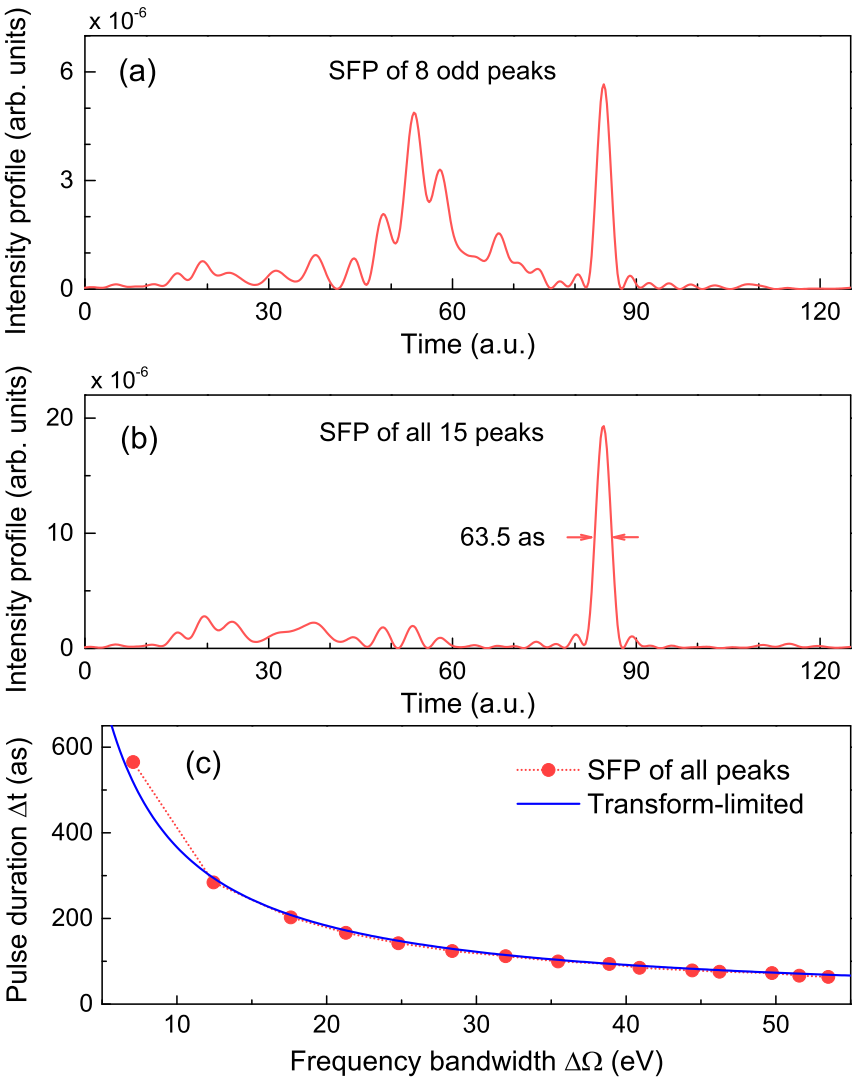

FIG. 10. (a) The striped-frequency pulse from eight odd peaks of the spectrum in Fig. 7. (b) The striped-frequency pulse from 15 odd and even peaks. (c) Plots of pulse duration $\Delta t$ as a function of the frequency bandwidth $\Delta \Omega$ for the transform-limited case of a square spectrum (solid line) and the SFP case of all peaks (dot-dash symbols).

short and long trajectories [25,37]. In general, high-harmonic radiation from long trajectories diverges more than that from short trajectories due to the more rapid variation of the dipole phase with the laser intensity which leads to a stronger curvature of the phase front $[51,52]$. We consider a gently focused beam such that the laser intensity variance within the gas medium is small. One can also adjust the gas target position [11,53] and collect HHG radiation off axis [54] so that both short and long trajectory radiations are phase matched. Moreover, our results show that in cases when short trajectory contributions are larger than long trajectory contributions (see, e.g., Fig. 9), one can still obtain SFPs.

\section{B. Three-dimensional intensity averaging}

When transverse averaging of the laser intensity cannot be ignored (e.g., the HHG detection area is large), we perform a $3 \mathrm{D}$ intensity averaging that includes both coherent longitudinal averaging and incoherent transverse averaging. In addition to summing spectral amplitudes along the laser propagating axis, we also integrate spectral intensities of the cross section at the medium exit:

$$
S_{3 \mathrm{D}}(\Omega)=\int_{0}^{h_{\max }} S_{1 \mathrm{D}}(\Omega, h) 2 \pi h d h,
$$

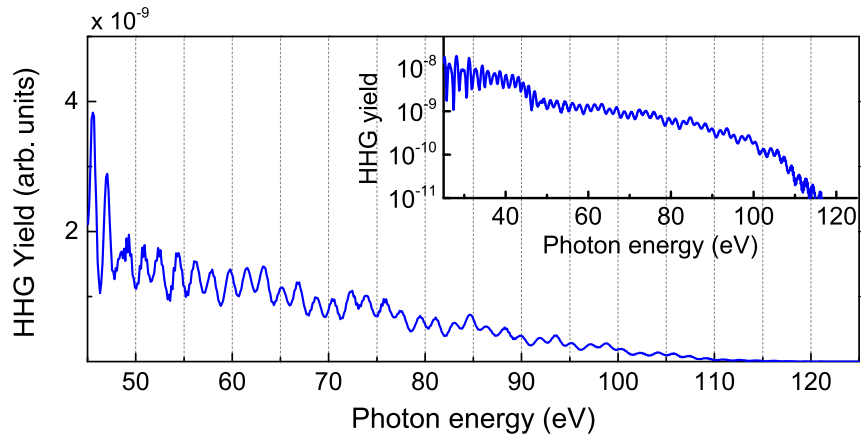

FIG. 11. TDSE HHG spectrum with 3D intensity averaging calculated using Eq. (16). The inset shows the same spectrum for a broader energy range on a log scale.

where $h$ is the radius of the cross section. The 1D spectrum $S_{1 \mathrm{D}}$ is evaluated as in Eq. (15) with a $4 \%$ variance in the laser intensity. $h_{\max }$ is chosen such that the laser intensity of a Gaussian beam drops to half of the center intensity, i.e., about $1 \times 10^{14} \mathrm{~W} / \mathrm{cm}^{2}$. Integration beyond such $h_{\max }$ does not change the HHG spectrum very much. Attosecond pulses are obtained by integrating 1D pulse intensities [see, e.g., Eq. (3) in Ref. [11]].

The TDSE HHG spectrum with 3D intensity averaging is shown in Fig. 11. The ordinary HHG harmonics with an equal spacing of $2 \omega_{0}$ in the low-energy plateau are still well resolved (see the spectrum below $50 \mathrm{eV}$ in the inset of Fig. 11). For the high-energy plateau above $50 \mathrm{eV}$, unlike the single-atom spectrum, Airy peaks are no longer visible and a supercontinuum structure appears. There is no definite cutoff for the high-energy plateau due to the wide range of laser intensities. For these reasons, we shall not present results for stripedfrequency pulses or a single spectral range near the cutoff. Note that Airy peaks in HHG spectra gradually disappear as we increase $h_{\max }$ in Eq. (16) and results shown in this section represent the worst averaging case.

We perform a two-dimensional attopulse duration scan for the supercontinuum spectrum in Fig. 11. The frequency bandwidth is chosen between $\Omega_{1}$ and $\Omega_{2}$ with $\Omega_{2}>\Omega_{1}$ where $\Omega_{1}$ is scanned from 48 to $99 \mathrm{eV}$ and $\Omega_{2}$ is scanned from 58 to $110 \mathrm{eV}$. The pulse duration is plotted in Fig. 12. There are three valleys in the contour plot and the lower left one in the low-frequency region exhibits the shortest pulse duration. The two edges of the triangle correspond to the 1D duration scan we have shown in Figs. 4(a) and 9(a): The left edge represents when $\Omega_{1}$ is fixed at the low-energy end $48 \mathrm{eV}$ and the top edge represents when $\Omega_{2}$ is fixed at the high-energy end $110 \mathrm{eV}$. Clearly, the minimum pulse generated from the low-frequency region (the valley on the lower left side) has a shorter duration than that from the high-frequency region (the valley on the upper right side).

The minimum attosecond pulses generated from lowfrequency and high-frequency regions are plotted in Fig. 13 where the low-frequency pulse has a bandwidth of $49-73 \mathrm{eV}$ and the high-frequency pulse has a bandwidth of $87-109 \mathrm{eV}$. Besides the shorter duration of 236 as as compared to 266 as, the minimum pulse generated from the low-frequency region is also more intense than that from the high-frequency region due to the higher HHG yield in the low-frequency region. 


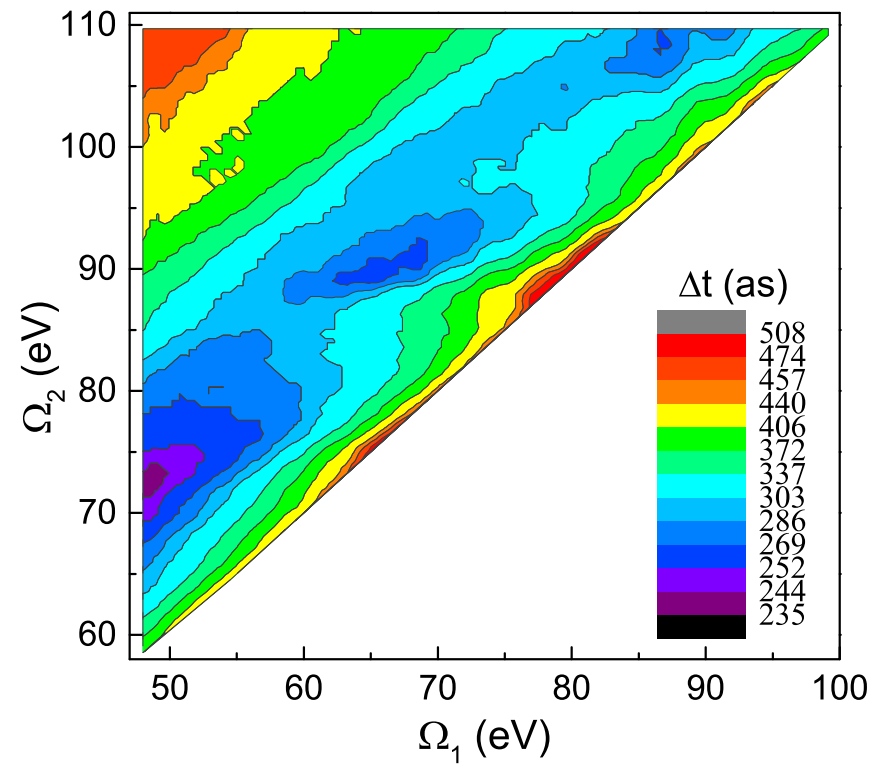

FIG. 12. Two-dimensional scan of attopulse duration $\Delta t . \Omega_{1}$ varies from 48 to $99 \mathrm{eV}$ and $\Omega_{2}$ varies from 58 to $110 \mathrm{eV}$.

\section{ANALYTIC ANALYSES OF SINGLE-ATOM RESULTS}

In this section, we analyze and explain our results about minimizing IAP duration through frequency selections of a one-cycle HHG spectrum based on single-atom calculations. Specifically, Sec. V A provides an approximation of the onecycle HHG spectral amplitude with an Airy function, which serves as a foundation for later analyses. Section V B analyzes results for a single spectral range near the cutoff by manually adjusting the cutoff Airy peak width. Section V C discusses striped-frequency pulses by coherently summing field amplitudes from individual Airy peaks at special times.

\section{A. Approximating HHG spectral amplitude with the Airy function}

First, we show that the spectral amplitude of a one-cycle HHG spectrum (meaning that only one laser cycle contributes to the HHG spectrum) can be approximated by an Airy function. For our analytic calculations of HHG, the field amplitude of the attosecond pulse is obtained by Fourier transforming the spectral dipole moment $D(\Omega)$ as given in Eq. (9). In general,

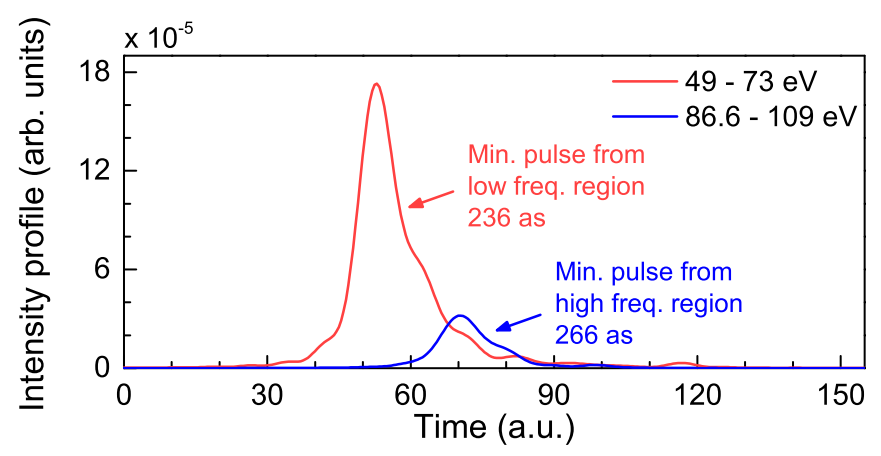

FIG. 13. Minimum attosecond pulses generated from low- and high-frequency regions of the HHG spectrum in Fig. 11. the spectral dipole moment $D(\Omega)$ is a coherent sum of halfcycle spectral amplitudes $\mathcal{A}_{j}(E)$ from each of the few cycles of a laser pulse [25,26]. For the main plateaus of the HHG spectra in Fig. 1, only one laser cycle contributes dominantly. In this case, the spectral dipole moment $D(\Omega)$ can be simply written as

$$
D(\Omega)=\eta \sqrt{\sigma^{(r)}} \operatorname{Ai}(x) e^{i\left(\phi+\Omega t_{r}\right)},
$$

where the factor $\eta$ and the phase $\phi$ are trajectory-related constants independent of $\Omega$, and $t_{r}$ is the return time of the cutoff trajectory, i.e., the trajectory with the maximum return energy. For the 1500-nm one-cycle pulse in our calculations, $t_{r}=85.5$ a.u. The $\operatorname{argument} x$ of the Airy function $\operatorname{Ai}(x)$ is a scaled dimensionless "energy":

$$
x=\frac{\hbar \Omega-E_{\mathrm{cut}}}{\epsilon E_{\mathrm{au}}}-1.019,
$$

where $E_{\text {cut }}$ is the cutoff energy and $E_{\text {au }}=|e|^{2} / a_{0}=27.21 \mathrm{eV}$ ( $a_{0}$ is the Bohr radius). Clearly, when the frequency $\Omega$ lies below the cutoff, the spectral dipole moment $D(\Omega)$ exhibits the oscillatory features of an Airy function which leads to the Airy peaks in the HHG spectrum. When $\Omega$ lies beyond the cutoff, the spectral dipole moment decays exponentially. $\epsilon$ is a dimensionless trajectory parameter which can be expressed as $[25,26]$

$$
\epsilon=\zeta^{1 / 3}, \quad \zeta=\frac{F\left(t_{r}\right)^{2}}{2 F_{\mathrm{au}}^{2}}\left[\frac{F\left(t_{r}\right)}{F\left(t_{i}\right)}-\frac{\dot{F}\left(t_{r}\right)}{F\left(t_{r}\right)}\left(t_{r}-t_{i}\right)-1\right] .
$$

Here $F(t)$ is the electric field in Eq. (13), $t_{i}$ is the ionization time of the cutoff trajectory, and $F_{\text {au }}=|e| / a_{0}^{2}=$ $5.142 \mathrm{GV} / \mathrm{cm}$. For the one-cycle pulse we use in our calculations, $\epsilon=0.132$. For simpler formulation, we also define a scaled dimensionless "time" $y$ :

$$
y=\frac{E_{\mathrm{au}}}{\hbar} \epsilon\left(t-t_{r}\right) .
$$

Substituting Eqs. (18) and (20) into Eqs. (17) and (9), we can approximate the attosecond-pulse field amplitude $d(t)$ as the Fourier transform of an Airy function $\tilde{d}(y)$ :

$$
d(t) \sim \epsilon \tilde{d}(y)=\epsilon \int_{-\infty}^{\infty} \operatorname{Ai}(x) \tilde{w}(x) e^{-i x y} d x .
$$

Notice that besides constant factors and overall phases, we also ignored the factor $\Omega^{2} \sqrt{\sigma^{(r)}}$ which has less pronounced variation with $\Omega$ compared to the oscillating Airy function in the HHG spectral range. Moreover, the phase factor $e^{i \Omega t_{r}}$ in Eq. (17) simply shifts the pulse in time. Therefore, in order to analyze attosecond pulses we can just focus on the Fourier transform of an Airy function $\tilde{d}(y)$ in Eq. (21).

To demonstrate that our analytic approximations are appropriate, we compare attosecond pulses generated from the TDSE spectrum [the solid line in Fig. 1(b)], the analytic spectrum [the dashed line in Fig. 1(b)], and the Airy function, which are calculated using Eqs. (7), (9), and (21), respectively. Figure 14(a) shows attosecond pulses obtained from a frequency bandwidth that includes the cutoff, 90-120 eV, of the TDSE and analytic spectra, and from the first four oscillations of an Airy peak. Pulses are scaled in intensity and the time of the Airy pulse is shifted according to Eq. (20). The TDSE pulse and the analytic pulse agree qualitatively that they 


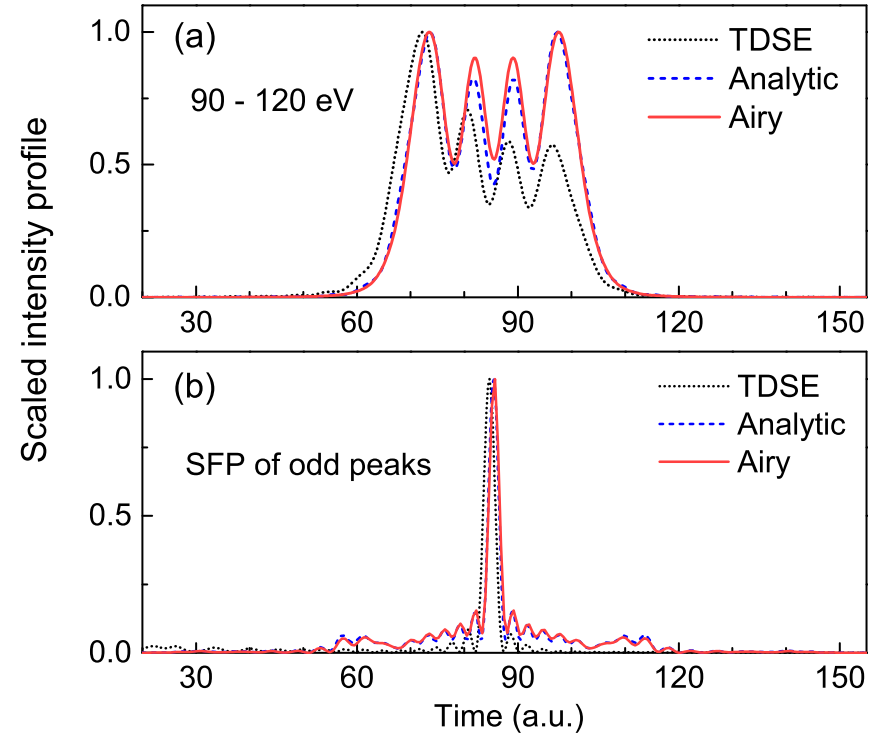

FIG. 14. Attosecond pulses generated from the TDSE spectrum (dotted lines), the analytic spectrum (dashed lines), and the Airy function (solid lines). Panel (a) is for a bandwidth of $90-120 \mathrm{eV}$ and panel (b) is for the SFP of odd peaks.

both produce one long pulse when the cutoff is included. The analytic pulse and the Airy pulse agree very well. Stripedfrequency pulses of odd peaks are shown in Fig. 14(b). In order to cover about the same bandwidth, the TDSE pulse is obtained from nine odd Airy peaks (1 through 17) of the TDSE spectrum, the analytic pulse is obtained from eight odd peaks (1 through 15) of the analytic spectrum, and the Airy pulse is obtained from the first eight odd oscillations of an Airy function. One sees that all three pulses agree very well.

\section{B. Cross-cutoff behavior and the cutoff Airy peak}

The cross-cutoff behavior of attosecond pulses that we see in Sec. III B where the pulse shape changes when including the cutoff is due to the fact that the cutoff Airy peak is spectrally much wider than other Airy peaks. This can be shown by manually adjusting the width of the cutoff peak. For example, one can approximate the cutoff peak of the spectral amplitude with a half sine wave $f_{1}$ :

$$
f_{1}(x)=a_{1} \sin \left(-b_{1} x+\frac{\pi}{4}\right) \text { for } x \in\left[-\frac{3 \pi}{4 b_{1}}, \frac{\pi}{4 b_{1}}\right],
$$

where $x$ is given by Eq. (18). The width of the cutoff peak can be then adjusted by varying the value of $b_{1}$. One sees in Figs. 15(a) and 15(b) that when $b_{1}=0.85$ the approximated cutoff peak $f_{1}$ can produce almost the exact attosecond pulse as that from the analytic HHG spectrum. When $b_{1}$ is increased such that the cutoff peak width is decreased to a comparable width as (or a smaller width than) the other Airy peaks, the cross-cutoff behavior disappears. For example, in Figs. 15(c) and $15(\mathrm{~d})$, when $b_{1}$ is increased to 1.35 and the cutoff peak has a comparable width as its neighbor, a pulse pair is obtained as the case for a single spectral range between cutoffs. On the other hand, when $b_{1}$ is decreased such that the cutoff peak is significantly much wider than the other Airy peaks,
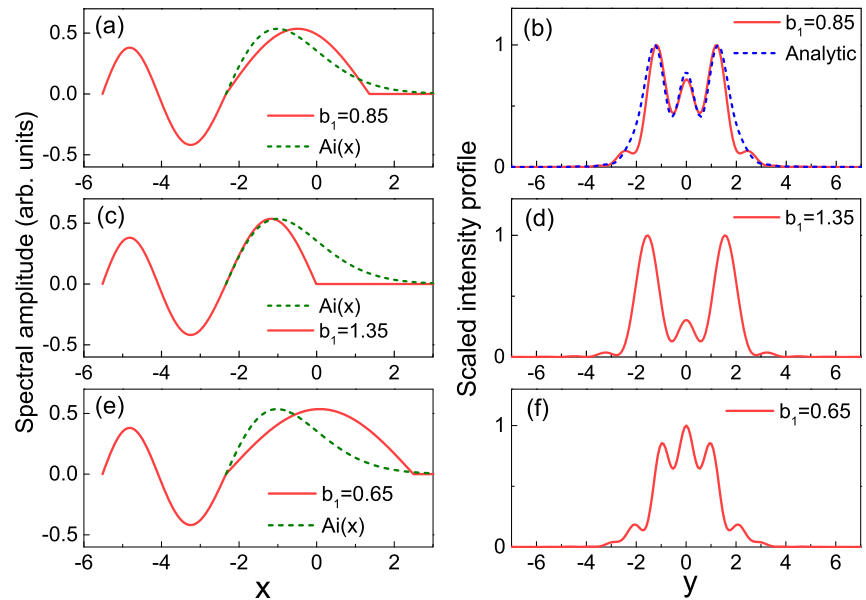

FIG. 15. (a, c, e) Solid (red) lines plot the approximated spectral amplitudes of the first three Airy peaks: the cutoff peak is described by $f_{1}$ in Eq. (22) and the second and third peaks are described by the Airy function $\operatorname{Ai}(x)$. The cutoff peak described by $\operatorname{Ai}(x)$ is plotted as dashed (green) lines for comparison. (b, d, f) Solid (red) lines plot attosecond pulses corresponding to spectral amplitudes (the solid lines) shown in panels (a), (c), and (e), respectively. The dashed (blue) line in panel (b) is the attosecond pulse obtained from the first three Airy peaks of the analytic HHG spectrum, which is then shifted in time according to Eq. (20).

the cross-cutoff behavior sustains. For example, in Figs. 15(e) and 15(f) when $b_{1}$ is decreased to 0.65 and the cutoff peak is significantly wider one obtains a single long pulse.

\section{Striped-frequency pulses}

We have seen that a half sine wave can describe the peak of the spectral amplitude very well. Indeed, the $n$th peak (or valley) of the spectral amplitude can be approximated by a half sine wave $f_{n}$ :

$$
f_{n}(x)=a_{n} \sin \left(-b_{n} x+\frac{\pi}{4}\right),
$$

where $x \in\left[-\pi / b_{n}(n-1 / 4),-\pi / b_{n}(n-5 / 4)\right]$ and $x$ is given by Eq. (18). Values of $a_{n}$ and $b_{n}$ can be obtained from, e.g., the Airy function:

$$
a_{n}=\frac{1}{\sqrt{\pi} \sqrt[4]{-\alpha_{n}}} \text { and } b_{n}=\frac{2}{3} \sqrt{-\alpha_{n}},
$$

where $\alpha_{n}$ are the zeros of $\operatorname{Ai}^{\prime}(x)$, i.e., $\operatorname{Ai}^{\prime}\left(\alpha_{n}\right)=0$.

Now the Fourier transform of each peak or valley of the spectral amplitude is simply the Fourier transform of a half sine wave, which yields

$$
\hat{f}_{n}(y)=\frac{a_{n} b_{n}}{y^{2}-b_{n}^{2}}\left[e^{i \frac{\pi}{b_{n}}\left(n-\frac{1}{4}\right) y}+e^{i \frac{\pi}{b_{n}}\left(n-\frac{5}{4}\right) y}\right] e^{i n \pi},
$$

where $y$ is given in Eq. (20). Each $\hat{f}_{n}$ is the individual field amplitude originated from the $n$th Airy peak in the HHG spectrum. We can obtain an attosecond-pulse field amplitude $\tilde{d}(y)$ by summing corresponding $\hat{f}_{n}$ 's:

$$
\tilde{d}(y) \approx \sum_{n} \hat{f}_{n}(y) .
$$


10

- Field amplitude for odd peaks

8

6

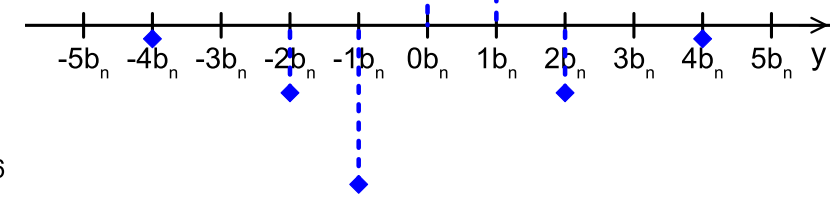

- Field amplitude

4 for even peaks

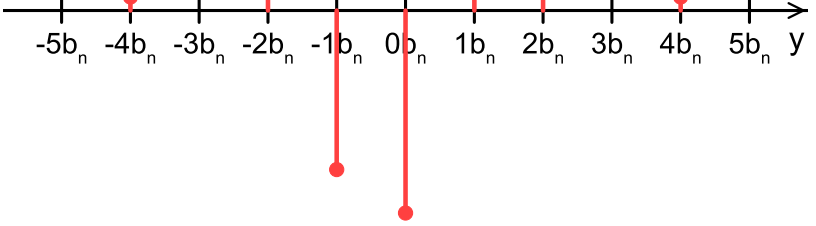

0

FIG. 16. Diagram of individual field amplitudes $\hat{f}_{n}$ at times of integer multiples of $b_{n}$ with locked phases [see Eq. (25)]. The height of the line-dot (dash-square) symbol is scaled to the magnitude of $\hat{f}_{n}$. For a striped-frequency pulse of odd (even) Airy peaks, one should add multiple copies of the odd-peak (even-peak) amplitude diagram [see Eq. (26)].

For example, to produce SFPs from odd or even Airy peaks, one can simply sum odd or even terms of $\hat{f}_{n}$.

The mechanism of generating SFPs can be understood by the phase structure of individual field amplitudes $\hat{f}_{n}$. In order for individual field amplitudes to add up constructively, they must have locked phases at a certain time, meaning that the phases of $\hat{f}_{n}$ are independent of $n$ at a certain time. One sees from Eq. (25) that at times of integer multiples of $b_{n}$, i.e., $y=$ $m b_{n}$ where $m \in \mathbb{Z}$, the phases of odd (even) amplitudes, i.e., $\hat{f}_{n}$ with odd (even) $n$ 's, are the same. Features of phase-locked individual field amplitudes are illustrated in Fig. 16, where the $m$-dependent phases are not shown.

At time $y=0$, i.e., at time $t=t_{r}$, individual field amplitudes $\hat{f}_{n}$ from odd (even) Airy peaks have exactly the same phase. Therefore, when selecting every other Airy peak in the HHG spectrum, individual field amplitudes add up constructively at time $t_{r}$ and produce a pulse peak with transform-limited duration.

Sidebands appear at times of integer multiples of $b_{n}$, i.e., $y=m b_{n}$, when individual field amplitudes also have lock phases at those times. However, owing to the attochirp in the HHG spectrum $b_{n}$ has different values for different Airy peaks $n$. Therefore, unlike the pulse peak at time $y=0$ which is inherently immune to the attochirp, sidebands from different Airy peaks are generated at different times and do not add up constructively when selecting many odd or even Airy peaks.
As a result of the above two aspects, i.e., the locked phase at time $t_{r}$ and the suppressed sidebands, a single attosecond pulse is produced with transform-limited duration when selecting every other Airy peak across the entire HHG spectrum.

\section{SUMMARY AND CONCLUSION}

Based on TDSE and analytic calculations of one-cycle HHG spectra (meaning that only one laser cycle contributes to the spectra) from an $\mathrm{H}$ atom produced by linearly polarized short laser pulses, we investigate ways to minimize the duration of an isolated attosecond pulse by selectively choosing frequencies in the HHG spectra without compensating the attochirp. One important feature of one-cycle HHG spectra is that they exhibit Airy peaks that come from subcycle interference of short and long trajectories, which is different from harmonic peaks with a $2 \omega_{0}$ spacing that come from intercycle interference.

From single-atom calculation results, we present strategies of producing shorter IAPs by careful frequency selections for three scenarios. When the high-frequency region of an HHG plateau is selected, one should use frequencies below the cutoff to produce shorter IAPs. For a wide HHG plateau where one cannot use the entire plateau bandwidth to produce short IAPs due to the attochirp, the low-frequency region can produce shorter IAPs than the high-frequency region. Lastly, one can obtain IAPs with transform-limited duration by selecting frequency stripes of every other Airy peak across the entire HHG plateau, or selecting all Airy peaks with a phase $\pi$ added to every other Airy peak. These striped-frequency pulses are the shortest (and strongest) attosecond pulses that can be obtained among the three scenarios. Analytic analyses show that how our frequency-selection strategies work is related to the (Fourier-transform) properties of an Airy function.

We also carry out HHG calculations considering macroscopic effects by means of intensity averaging over the focal region. We find that when laser intensity variance in the focal region is small such that macroscopic HHG spectra resemble key features of single-atom spectra, i.e., the Airy peaks, our strategies for minimizing IAPs can still apply. When macroscopic HHG spectra do not exhibit Airy peaks (but a supercontinuum), only one strategy is applicable that the low-frequency region can produce shorter IAPs than the high-frequency region.

Our studies provide methods to minimize IAP duration especially for a broad one-cycle HHG spectrum. Since the HHG spectrum bandwidth scales roughly quadratically with the driving laser wavelength [28,29], application of emerging long-wavelength driving lasers can generate HHG spectra with broader bandwidths. Our methods take advantage of new laser systems with ultrashort pulse duration and long driving wavelengths in generating short and bright attosecond pulses effectively.

\section{ACKNOWLEDGMENTS}

This work was supported by the US National Science Foundation under Grant No. PHYS-1505492. Our TDSE calculations were completed utilizing the Holland Computing Center of the University of Nebraska, which receives support 
from the Nebraska Research Initiative. The authors gratefully acknowledge M. Fuchs for insightful discussions regarding the experimental design, and M. Fuchs and A. W. Voshall for helpful suggestions regarding writing this paper.
[1] F. Krausz and M. Ivanov, Attosecond physics, Rev. Mod. Phys. 81, 163 (2009).

[2] M. Chini, K. Zhao, and Z. Chang, The generation, characterization and applications of broadband isolated attosecond pulses, Nature Photon 8, 178 (2014).

[3] F. Calegari, G. Sansone, S. Stagira, C. Vozzi, and M. Nisoli, Advances in attosecond science, J. Phys. B 49, 062001 (2016).

[4] M. Nisoli, P. Decleva, F. Calegari, A. Palacios, and F. Martín, Attosecond electron dynamics in molecules, Chem. Rev. 117, 10760 (2017).

[5] M. F. Ciappina, J. A. Pérez-Hernández, A. S. Landsman, W. A. Okell, S. Zherebtsov, B. Förg, J. Schötz, L. Seiffert, T. Fennel, T. Shaaran, T. Zimmermann, A. Chacón, R. Guichard, A. Zaï, J. W. G. Tisch, J. P. Marangos, T. Witting, A. Braun, S. A. Maier, L. Roso, M. Krüger, P. Hommelhoff, M. F. Kling, F. Krausz, and M. Lewenstein, Attosecond physics at the nanoscale, Rep. Prog. Phys. 80, 054401 (2017).

[6] P. M. Kraus, M. Zürch, S. K. Cushing, D. M. Neumark, and S. R. Leone, The ultrafast x-ray spectroscopic revolution in chemical dynamics, Nat. Rev. Chem. 2, 82 (2018).

[7] E. Goulielmakis, M. Schultze, M. Hofstetter, V. S. Yakovlev, J. Gagnon, M. Uiberacker, A. L. Aquila, E. M. Gullikson, D. T. Attwood, R. Kienberger, F. Krausz, and U. Kleineberg, Singlecycle nonlinear optics, Science 320, 1614 (2008).

[8] K. Zhao, Q. Zhang, M. Chini, Y. Wu, X. Wang, and Z. Chang, Tailoring a 67 attosecond pulse through advantageous phasemismatch, Opt. Lett. 37, 3891 (2012).

[9] T. Gaumnitz, A. Jain, Y. Pertot, M. Huppert, I. Jordan, F. Ardana-Lamas, and H. J. Wörner, Streaking of 43-attosecond soft-x-ray pulses generated by a passively CEP-stable midinfrared driver, Opt. Express 25, 27506 (2017).

[10] M. T. Hassan, T. T. Luu, A. Moulet, O. Raskazovskaya, P. Zhokhov, M. Garg, N. Karpowicz, A. M. Zheltikov, V. Pervak, F. Krausz, and E. Goulielmakis, Optical attosecond pulses and tracking the nonlinear response of bound electrons, Nature (London) 530, 66 (2016).

[11] P. Antoine, A. L'Huillier, and M. Lewenstein, Attosecond Pulse Trains Using High-Order Harmonics, Phys. Rev. Lett. 77, 1234 (1996).

[12] P. M. Paul, E. S. Toma, P. Breger, G. Mullot, F. Auge, P. Balcou, H. G. Muller, and P. Agostini, Observation of a train of attosecond pulses from high harmonic generation, Science 292, 1689 (2001).

[13] I. P. Christov, M. M. Murnane, and H. C. Kapteyn, HighHarmonic Generation of Attosecond Pulses in the "SingleCycle" Regime, Phys. Rev. Lett. 78, 1251 (1997).

[14] M. Hentschel, R. Kienberger, C. Spielmann, G. A. Reider, N. Milosevic, T. Brabec, P. Corkum, U. Heinzmann, M. Drescher, and F. Krausz, Attosecond metrology, Nature (London) 414, 509 (2001).

[15] A. Baltuška, T. Udem, M. Uiberacker, M. Hentschel, E. Goulielmakis, C. Gohle, R. Holzwarth, V. S. Yakovlev, A. Scrinzi, T. W. Hänsch, and F. Krausz, Attosecond control of electronic processes by intense light fields, Nature (London) 421, 611 (2003).

[16] G. Sansone, E. Benedetti, F. Calegari, C. Vozzi, L. Avaldi, R. Flammini, L. Poletto, P. Villoresi, C. Altucci, R. Velotta, S. Stagira, S. De Silvestri, and M. Nisoli, Isolated single-cycle attosecond pulses, Science 314, 443 (2006).

[17] R. Kienberger, E. Goulielmakis, M. Uiberacker, A. Baltuska, V. Yakovlev, F. Bammer, A. Scrinzi, T. Westerwalbesloh, U. Kleineberg, U. Heinzmann, M. Drescher, and F. Krausz, Atomic transient recorder, Nature (London) 427, 817 (2004).

[18] M.-C. Chen, C. Mancuso, C. Hernández-García, F. Dollar, B. Galloway, D. Popmintchev, P.-C. Huang, B. Walker, L. Plaja, A. A. Jaroń-Becker, A. Becker, M. M. Murnane, H. C. Kapteyn, and T. Popmintchev, Generation of bright isolated attosecond soft x-ray pulses driven by multicycle midinfrared lasers, Proc. Natl. Acad. Sci. USA 111, E2361 (2014).

[19] T. Popmintchev, M.-C. Chen, D. Popmintchev, P. Arpin, S. Brown, S. Ališauskas, G. Andriukaitis, T. Balčiunas, O. D. Mücke, A. Pugzlys, A. Baltuška, B. Shim, S. E. Schrauth, A. Gaeta, C. Hernández-García, L. Plaja, A. Becker, A. JaronBecker, M. M. Murnane, and H. C. Kapteyn, Bright coherent ultrahigh harmonics in the $\mathrm{keV} x$-ray regime from mid-infrared femtosecond lasers, Science 336, 1287 (2012).

[20] Y. Mairesse, A. de Bohan, L. J. Frasinski, H. Merdji, L. C. Dinu, P. Monchicourt, P. Breger, M. Kovačev, R. Taïeb, B. Carré, H. G. Muller, P. Agostini, and P. Salières, Attosecond synchronization of high-harmonic soft x-rays, Science 302, 1540 (2003).

[21] Z. Chang, Chirp of the single attosecond pulse generated by a polarization gating, Phys. Rev. A 71, 023813 (2005).

[22] R. López-Martens, K. Varjú, P. Johnsson, J. Mauritsson, Y. Mairesse, P. Salières, M. B. Gaarde, K. J. Schafer, A. Persson, S. Svanberg, C.-G. Wahlström, and A. L'Huillier, Amplitude and Phase Control of Attosecond Light Pulses, Phys. Rev. Lett. 94, 033001 (2005).

[23] I.-L. Liu, P.-C. Li, and S.-I. Chu, Coherent control of the electron quantum paths for the generation of single ultrashort attosecond laser pulse, Phys. Rev. A 84, 033414 (2011).

[24] X.-M. Tong and S.-I. Chu, Theoretical study of multiple high-order harmonic generation by intense ultrashort pulsed laser fields: A new generalized pseudospectral time-dependent method, Chem. Phys. 217, 119 (1997).

[25] M. V. Frolov, N. L. Manakov, A. M. Popov, O. V. Tikhonova, E. A. Volkova, A. A. Silaev, N. V. Vvedenskii, and A. F. Starace, Analytic theory of high-order-harmonic generation by an intense few-cycle laser pulse, Phys. Rev. A 85, 033416 (2012).

[26] D. Peng, L.-W. Pi, M. V. Frolov, and A. F. Starace, Enhancing high-order-harmonic generation by time delays between twocolor, few-cycle pulses, Phys. Rev. A 95, 033413 (2017).

[27] K. J. Schafer, B. Yang, L. F. DiMauro, and K. C. Kulander, Above Threshold Ionization Beyond the High Harmonic Cutoff, Phys. Rev. Lett. 70, 1599 (1993). 
[28] K. C. Kulander, K. J. Schafer, and J. L. Krause, Dynamics of Short-Pulse Excitation, Ionization and Harmonic Conversion, in Super-Intense Laser-Atom Physics, edited by B. Piraux, A. L'Huillier, and K. Rzaążewski (Plenum, New York, 1993), pp. 95-110.

[29] P. B. Corkum, Plasma Perspective on Strong Field Multiphoton Ionization, Phys. Rev. Lett. 71, 1994 (1993).

[30] M. Lewenstein, P. Balcou, M. Y. Ivanov, A. L'Huillier, and P. B. Corkum, Theory of high-harmonic generation by lowfrequency laser fields, Phys. Rev. A 49, 2117 (1994).

[31] A. D. Bandrauk, S. Chelkowski, D. J. Diestler, J. Manz, and K.-J. Yuan, Quantum simulation of high-order harmonic spectra of the hydrogen atom, Phys. Rev. A 79, 023403 (2009).

[32] D. Peng, M. V. Frolov, L.-W. Pi, and A. F. Starace, Enhancing high-order harmonic generation by sculpting waveforms with chirp, Phys. Rev. A 97, 053414 (2018).

[33] R.-F. Lu, H.-X. He, Y.-H. Guo, and K.-L. Han, Theoretical study of single attosecond pulse generation with a three-colour laser field, J. Phys. B 42, 225601 (2009).

[34] A. de Bohan, P. Antoine, D. B. Milošević, and B. Piraux, PhaseDependent Harmonic Emission with Ultrashort Laser Pulses, Phys. Rev. Lett. 81, 1837 (1998).

[35] M. Nisoli, G. Sansone, S. Stagira, S. De Silvestri, C. Vozzi, M. Pascolini, L. Poletto, P. Villoresi, and G. Tondello, Effects of Carrier-Envelope Phase Differences of Few-Optical-Cycle Light Pulses in Single-Shot High-Order-Harmonic Spectra, Phys. Rev. Lett. 91, 213905 (2003).

[36] C. A. Haworth, L. E. Chipperfield, J. S. Robinson, P. L. Knight, J. P. Marangos, and J. W. G. Tisch, Half-cycle cutoffs in harmonic spectra and robust carrier-envelope phase retrieval, Nat. Phys. 3, 52 (2007).

[37] D. B. Milošević and W. Becker, Role of long quantum orbits in high-order harmonic generation, Phys. Rev. A 66, 063417 (2002).

[38] Z. Chang, Single attosecond pulse and XUV supercontinuum in the high-order harmonic plateau, Phys. Rev. A 70, 043802 (2004).

[39] J. Tate, T. Auguste, H. G. Muller, P. Salières, P. Agostini, and L. F. DiMauro, Scaling of Wave-Packet Dynamics in an Intense Midinfrared Field, Phys. Rev. Lett. 98, 013901 (2007).

[40] P.-C. Li, C. Laughlin, and S.-I. Chu, Generation of isolated sub20-attosecond pulses from He atoms by two-color midinfrared laser fields, Phys. Rev. A 89, 023431 (2014).

[41] A. M. Weiner, Femtosecond pulse shaping using spatial light modulators, Rev. Sci. Instrum. 71, 1929 (2000).

[42] I. J. Sola, E. Mével, L. Elouga, E. Constant, V. Strelkov, L. Poletto, P. Villoresi, E. Benedetti, J.-P. Caumes, S. Stagira, C. Vozzi, G. Sansone, and M. Nisoli, Controlling attosecond electron dynamics by phase-stabilized polarization gating, Nat. Phys. 2, 319 (2006).

[43] W. Schweinberger, A. Sommer, E. Bothschafter, J. Li, F. Krausz, R. Kienberger, and M. Schultze, Waveform-controlled near-single-cycle milli-joule laser pulses generate sub-10 nm extreme ultraviolet continua, Opt. Lett. 37, 3573 (2012).

[44] D. E. Rivas, A. Borot, D. E. Cardenas, G. Marcus, X. Gu, D. Herrmann, J. Xu, J. Tan, D. Kormin, G. Ma, W. Dallari, G. D. Tsakiris, I. B. Földes, S.-w. Chou, M. Weidman, B. Bergues, T. Wittmann, H. Schröder, P. Tzallas, D. Charalambidis, O. Razskazovskaya, V. Pervak, F. Krausz, and L. Veisz, Next generation driver for attosecond and laser-plasma physics, Sci. Rep. 7, 5224 (2017).

[45] A. L'Huillier, K. J. Schafer, and K. C. Kulander, Theoretical aspects of intense field harmonic generation, J. Phys. B 24, 3315 (1991).

[46] P. Balcou, P. Sali ‘eres, A. L'Huillier, and M. Lewenstein, Generalized phase-matching conditions for high harmonics: The role of field-gradient forces, Phys. Rev. A 55, 3204 (1997).

[47] E. Priori, G. Cerullo, M. Nisoli, S. Stagira, S. De Silvestri, P. Villoresi, L. Poletto, P. Ceccherini, C. Altucci, R. Bruzzese, and C. de Lisio, Nonadiabatic three-dimensional model of highorder harmonic generation in the few-optical-cycle regime, Phys. Rev. A 61, 063801 (2000).

[48] T. Morishita, A. T. Le, Z. Chen, and C. D. Lin, Accurate Retrieval of Structural Information from Laser-Induced Photoelectron and High-Order Harmonic Spectra by Few-Cycle Laser Pulses, Phys. Rev. Lett. 100, 013903 (2008).

[49] C. Jin, A. T. Le, and C. D. Lin, Retrieval of target photorecombination cross sections from high-order harmonics generated in a macroscopic medium, Phys. Rev. A 79, 053413 (2009).

[50] M. Tudorovskaya and M. Lein, High-order harmonic generation in the presence of a resonance, Phys. Rev. A 84, 013430 (2011).

[51] M. Bellini, C. Lyngå, A. Tozzi, M. B. Gaarde, T. W. Hänsch, A. L'Huillier, and C.-G. Wahlström, Temporal Coherence of Ultrashort High-Order Harmonic Pulses, Phys. Rev. Lett. 81, 297 (1998).

[52] P. Salières, A. L'Huillier, and M. Lewenstein, Coherence Control of High-Order Harmonics, Phys. Rev. Lett. 74, 3776 (1995).

[53] P. Salières, B. Carré, L. Le Déroff, F. Grasbon, G. G. Paulus, H. Walther, R. Kopold, W. Becker, D. B. Milosević, A. Sanpera, and M. Lewenstein, Feynman's path-integral approach for intense-laser-atom interactions, Science 292, 902 (2001).

[54] A. Zaïr, M. Holler, A. Guandalini, F. Schapper, J. Biegert, L. Gallmann, U. Keller, A. S. Wyatt, A. Monmayrant, I. A. Walmsley, E. Cormier, T. Auguste, J. P. Caumes, and P. Salières, Quantum Path Interferences in High-Order Harmonic Generation, Phys. Rev. Lett. 100, 143902 (2008). 